\title{
Research on Credit Risk Measurement Based on Uncertain KMV Model
}

\author{
Ni Zhan, Liang Lin, Ting Lou \\ College of Science, Guilin University of Technology, Guilin, China \\ Email: zhanni105@163.com
}

Received May 25, 2013; revised June 25, 2013; accepted July 15, 2013

Copyright (C) 2013 Ni Zhan et al. This is an open access article distributed under the Creative Commons Attribution License, which permits unrestricted use, distribution, and reproduction in any medium, provided the original work is properly cited.

\begin{abstract}
Regarding KMV model identification credit risk profile of small and medium-sized listed companies, at present, domestic scholars has made some achievements in the process of the KMV model combined with China's national conditions. In this paper, we will amend the model by using uncertain interest rate instead of fixed rate on the basis of existing research. Comparing the uncertain KMV model to traditional KMV model with ST-listed companies and nonST-listed companies in Shanghai and Shenzhen stock exchange, we find that it performs slightly better as a predictor in uncertain KMV model and in out of sample forecasts.
\end{abstract}

Keywords: Credit Risks; KMV Model; Uncertain Interest Rate

\section{Introduction}

With the improvement of the activeness in economic activities and enhancement of national economic relationships between countries, people found that it's important and necessary to be on guard and control the various risks in economic activities. Barings Bank event as well as a series of major financial institutions crisis since the mid-1990s of the $20^{\text {th }}$ century, makes the Banks increasingly concerned about the risk prevention, measurement and management. Nowadays commercial banks face two kinds of risk, one is non-systemic risk, such as credit risk, settlement risk, and the other is systemic risk, such as market risk, interest rate risk, currency risk and so on, and credit risk is the main risk in that commercial banks face in the course of business. And how to guard against and reduce credit risk is an urgent requirement to the commercial banks.

W. Zhang [1] carried out a theoretical study of the credit risk of listed companies measurement and analyzed the samples of China's listed companies. Research shows that at this stage, KMV model based on options pricing breach theory could well apply to China's credit risk measurement of listed companies. Z. J. Zhang \& X. H. Chen [2] set two credit warning lines to monitor the credit crisis of small and medium-sized listed companies by KMV model. X. Y. Liang [3] made the empirical analysis of KMV model widely used in foreign countries combined with the actual situation in China, and they amended parameters accordingly in order to enhance the applicability of the model in the Chinese market. S. C. Yang [4] used qualitative and demonstration method to carry on the theoretical analysis and empirical research on credit risk measure model, furthermore, he made analysis on KMV model used in Chinese enterprise with newest data of corporation in stock market.

Most of the existing credit risk measurement methods are "static" models by using risk-free rate to estimate the uncertain risk. As we all know, interest rate in real life is uncertain. In order to reflect and solve practical problems better, we will introduce the uncertain variable into the research of credit risk.

\section{KMV Model Theory}

\subsection{The Basic Assumptions of the Model}

KMV model basic idea is that viewed the company equity as a European call options, viewed market value of assets as the subject, the nominal value of company's debt as exercise price. Company will choose to repay the debt when market value of assets is greater than the value of the debt, default while market value of assets is less than the value of debt.

1) Satisfy the basic assumptions of the Merton model: the company's stock price is a random process, transac- 
tion is no friction, etc., and the change of enterprise's value obeys Ito Process.

2) Company will default to its creditors and shareholders when the company's asset value falls below a certain level.

3) The capital structure of the borrower only includes owner's equity, short-term debt, long-term debt and convertible preferred stock.

\subsection{The KMV Calculated Steps}

1) The Asset Value and Volatility

If $V_{E}$ represents market value of equity, $V_{A}$ represents firm's asset value, then the relationship between the companies' equity and asset value can be expressed as

$$
V_{E}=V_{A} N\left(d_{1}\right)-\mathrm{e}^{-r T} D N\left(d_{2}\right)
$$

where $D$ represents book value of debt which is due at time $T, r$ is the risk free interest rate, $N(\cdot)$ is the cumulative standard normal distribution function, $d_{1}$ and $d_{2}$ are given by

$$
\begin{aligned}
& d_{1}=\frac{\ln \left(\frac{V_{A}}{D}\right)+\left(r+\frac{1}{2} \sigma_{A}^{2}\right)}{\sigma_{A} \sqrt{T}} \\
& d_{2}=d_{1}-\sigma_{A} \sqrt{T}
\end{aligned}
$$

Equity volatility and asset volatility relationship:

$$
\sigma_{E}=\frac{V_{A} N\left(d_{1}\right)}{V_{E}} \sigma_{A}
$$

where $\sigma_{E}$ is equity volatility, $\sigma_{A}$ is asset volatility.

The asset value and volatility implied by the equity value, equity volatility and liabilities are calculated by solving the call price and volatility equations, (1) and (4), simultaneously.

2) Default to Distance

The default point (DPT) is generally determined by the short-term liabilities and long-term liabilities:

$$
\mathrm{DPT}=\mathrm{STD}+\frac{1}{2} \mathrm{LTD}
$$

Distance to default (DD) is the relative distance between the future value of the assets and default point. Once this numerical solution is obtained, the distance to default can be calculated as

$$
\mathrm{DD}=\frac{V_{A}-\mathrm{DPT}}{V_{A} \sigma_{A}}
$$

\section{3) EDF (Expected Default Frequency)}

The theoretical value of expected default frequency is based on the assumption that market value of the assets follows a normal distribution,

$$
\mathrm{EDF}=N(-\mathrm{DD})
$$

It still lacks large amounts of data resources to establish an effective database in China, so the empirical section, we select the distance to default to measure credit risk. The greater the distance to default, the smaller the likelihood of default, the company's credit status is better; on the contrary, the smaller the distance to default, the higher the risk of default.

The vast majority of scholars simply assumed $r$ is a free-rate and did not give a reasonable explanation, but we all know interest rate in real life is uncertain, we will introduce the uncertain variable into the research of credit risk.

\section{Uncertainty KMV Model}

Uncertainty theory was founded by Liu [5] in 2007 and refined by Liu [6] in 2010, which is a branch of mathematics based on normality, monotonicity, self-duality, countable subadditivity, and product measure axioms.

\subsection{Uncertain Measure and Uncertain Variable}

Let $\Gamma$ be a nonempty set, and $\mathcal{L}$ a $\sigma$-algebra over $\Gamma$. Each element $\Lambda \in \mathcal{L}$ is called an event. Uncertain measure $\mathcal{M}$ was introduced as a set function satisfying the following five axioms [5]:

Axiom 1. (Normality Axiom) $\mathcal{M}\{\Gamma\}=1$ for the universal set $\Gamma$.

Axiom 2. (Monotonicity Axiom) $\mathcal{M}\left\{\Gamma_{1}\right\} \leq \mathcal{M}\left\{\Gamma_{2}\right\}$ whenever $\Lambda_{1} \subset \Lambda_{2}$.

Axiom 3. (Self-Duality Axiom) $\mathcal{M}\{\Lambda\}+\mathcal{M}\left\{\Lambda^{c}\right\}=1$ for any event $\Lambda$.

Axiom 4. (Countable Subadditivity Axiom) For every countable sequence of events $\left\{\Lambda_{i}\right\}$, we have

$$
\mathcal{M}\left\{\bigcup_{i=1}^{\infty} \Lambda_{i}\right\} \leq \sum_{i=1}^{\infty} \mathcal{M}\left\{\Lambda_{i}\right\} .
$$

Axiom 5. (Product Measure Axiom) Let $\Gamma_{k}$ be nonempty sets on which $\mathcal{M}_{k}$ are uncertain measures $k=1,2, \cdots, n$, respectively. Then the product uncertain measure $\mathcal{M}$ is an uncertain measure on the product $\sigma$-algebra $\mathcal{L}_{1} \times \mathcal{L}_{2} \times \cdots \times \mathcal{L}_{n}$ satisfying

$$
\mathcal{M}\left\{\prod_{k=1}^{n} \Lambda_{k}\right\} \leq \min _{1 \leq k \leq n} \mathcal{M}_{k}\left\{\Lambda_{k}\right\} .
$$

where $\Lambda_{k} \in \mathcal{L}_{k}, k=1,2, \cdots, n$.

Definition 3.1.1 (Liu [5]) Let $\Gamma$ be a nonempty set, and $\mathcal{L}$ be a $\sigma$-algebra over $\Gamma$, and $\mathcal{M}$ an uncertain measure. Then the triplet $(\Gamma, \mathcal{L}, \mathcal{M})$ is called an uncertainty space.

Definition 3.1.2 (Liu [5]) An uncertain variable is a measurable function from an uncertainty space $(\Gamma, \mathcal{L}, \mathcal{M})$ to the set of real numbers. 


\subsection{Uncertain Distribution and Inverse Uncertain Distribution}

Definition 3.2.1 [6] The uncertainty distribution $\Phi$ of an uncertain variable $\xi$ is defined by

$$
\Phi(x)=M\{\xi \leq x\}
$$

Definition 3.2.2 [6]: An uncertain variable $\xi$ is called linear if it has a linear uncertainty distribution

$$
\Phi(x)=\left\{\begin{array}{lc}
0, & x<a \\
(x-a) /((b-a), & a \leq x \leq b \\
1, & x>b
\end{array}\right.
$$

denoted by $\mathcal{L}(a, b)$ where a and b are real numbers with $a<b$. Distribution shown in Figure 1.

Definition 3.2.3 [6]: An uncertain variabe $\xi$ is called normal if it has a normal uncertainty distribution

$$
\Phi(x)=\left(1+\exp \left(\frac{\pi(e-x)}{\sqrt{3} \sigma}\right)\right)^{-1}
$$

denoted by $\mathcal{N}(e, \sigma)$ where $e$ and $\sigma$ are real numbers with $\sigma>0$. Distribution shown in Figure 2 .

Definition 3.2.4 (Liu [6]) An uncertainty distribution $\Phi$ is call lognormal if $\ln \xi$ is a normal uncertain variable $\mathcal{N}(e, \sigma)$. In other words, a lognormal uncertain variable has an uncertainty distribution

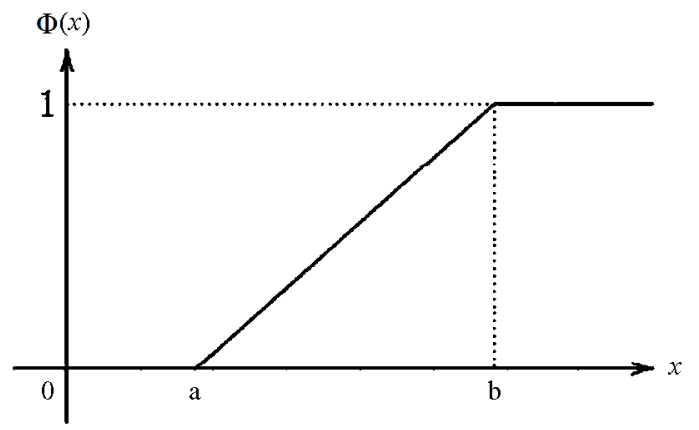

Figure 1. A linear uncertainty distribution.

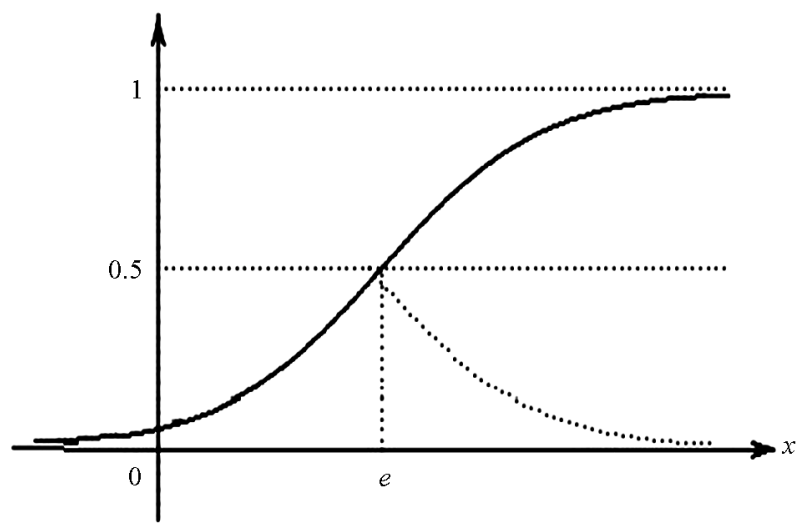

Figure 2. A normal uncertainty distribution.

$$
\Phi(x)=\left(1+\exp \left(\frac{\pi(e-\ln x)}{\sqrt{3} \sigma}\right)\right)^{-1}, x>0
$$

denoted by $\mathcal{L} \mathcal{O G N}(e, \sigma)$, where $e$ and $\sigma$ are real numbers with $\sigma>0$. Distribution shown in Figure 3.

Definition 3.2.5 (Liu [6])An uncertainty distribution $\Phi$ is said to be regular if its inverse function $\Phi^{-1}(\alpha)$ exists and is unique for each $\alpha \in(0,1)$.

As we all know, the firm's asset value is a decreasing function of interest $r$ which is adjusted irregularly [7]. Let $r$ be an uncertain variable with regular lognormal uncertainty distribution $\Phi$. Then the inverse function $\Phi^{-1}$ is called the inverse uncertainty distribution of $r$. It's easy to verify that the inverse uncertainty distribution of lognormal uncertain variable is:

$$
\Phi^{-1}(\alpha)=\exp (e)\left(\frac{\alpha}{1-\alpha}\right)^{\sqrt{3} \sigma / \pi}=r
$$

\section{Numerical Examples}

In this paper, we choose 6 ST and *ST-listed companies, which are added ST and ${ }^{*}$ ST in 2011, and 6 non-STlisted companies in Shanghai and Shenzhen stock exchange as empirical research samples, in which ST and ${ }^{*}$ ST companies are considered as default samples while non-ST companies are considered as normal samples. Data is from http://finance.sina.com.cn/.

After finishing the row data, we get $V_{E}$ and $\sigma_{E}$ shown in Table 1. Compare the traditional KMV model which uses a fixed interest rate and uncertain KMV model by using the above data.

\subsection{Traditional KMV Model}

We select deposit interest rate-3.5\% at the end of 2011, put $V_{E}$ and $\sigma_{E}$ into formulas (1)-(4), and obtain each listed company's market value of asset and asset volatility by the iterate program of MATLAB as shown in Ta-

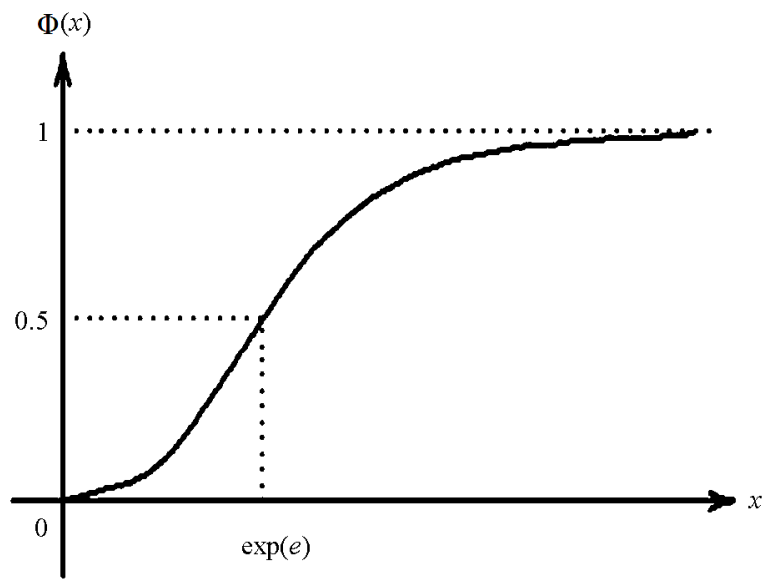

Figure 3. A lognormal uncertainty distribution. 
ble 2. Put the data in Table 2 into (6) obtain default to distance, as shown in Table 3.

From Table 3, we find that part of the ${ }^{*}$ ST and ST companies' DD are bigger than non-ST companies which may lead to an evaluation error.

\subsection{Uncertain KMV Model}

Let $r$ be an uncertain variable with normal uncertainty distribution and can be obtain by Delphi method. The consultation process is as follows:

Q1: What do you think is the minimum interest rate?

A1: 0.02. (an expert's experimental data $(0.02,0.3)$ is acquired).

Q2: What do you think is the maximum interest rate?

A2: 0.05. (an expert's experimental data $(0.05,1)$ is acquired).

Q3: What do you think is a likely interest rate?

A3: 0.03. (an expert's experimental data $(0.035,0.75)$ is acquired)).

Q4: What is the belief degree that the real interest rate is less than 0.04 ?

A4: 0.8. (an expert's experimental data $(0.04,0.8)$ is acquired)).

Q5: What is the belief degree that the real interest rate is less than 0.045 ?

A5: 0.9. (an expert's experimental data $(0.045,0.9)$ is acquired)).

Where $(0.02,0.3),(0.035,0.75),(0.04,0.8),(0.045$, $0.9),(0.05,1)$ represents

$\Phi(0.02)=\mathcal{M}(\xi \leq 0.02)=0.3$,

$\Phi(0.05)=\mathcal{M}(\xi \leq 0.05)=1$,

$\Phi(0.035)=\mathcal{M}(\xi \leq 0.03)=0.75$,

$\Phi(0.04)=\mathcal{M}(\xi \leq 0.04)=0.8$,

$\Phi(0.045)=\mathcal{M}(\xi \leq 0.045)=0.9$, respectively.

So we obtain the inverse uncertainty distribution of lognormal uncertain variable by using expert's experimental data:

$$
\Phi^{-1}(\alpha)=\exp (-3.89)\left(\frac{\alpha}{1-\alpha}\right)^{-0.747}=r
$$

We get DD by using uncertainty interest rate shown in Table 4:

Compare $\mathrm{DD}_{1}$ and $\mathrm{DD}_{2}$ in the same drawing.

From Figure 4, we can find that, for ${ }^{*}$ ST and ST companies, calculations of uncertain KMV model are smaller than traditional KMV model's; for non-ST-companies, calculations of uncertain KMV model are bigger than traditional KMV model's. By this example, it is verified

Table 1. Equity value and equity volatility.

\begin{tabular}{cccc}
\hline & Equity Value $V_{E}$ (Ten thousand) & Equity volatility $\sigma_{E}$ & Debt D (Ten thousand) \\
\hline${ }^{*}$ ST Yuancheng & 100094.06 & 0.40924 & 47636.68 \\
${ }^{*}$ ST Zhongda & 302186.68 & 0.45036 & 244120.24 \\
${ }^{*}$ ST Tianrun & 123571.38 & 0.52767 & 27944.7 \\
ST Guofa & 178142.36 & 0.44814 & 48128.76 \\
ST Luodun & 229618.29 & 0.58704 & 56683.21 \\
ST Tianyi & 103130.9 & 0.41686 & 41352.81 \\
Jinma & 240603 & 0.42448 & 63062.24 \\
Lianchuang & 460546.02 & 0.51624 & 75134.07 \\
Kaile & 364071.6 & 0.38353 & 181406.34 \\
Batian & 194009.26 & 0.53973 & 12566.91 \\
Jiahua & 1358366 & 0.3553 & 60442.38 \\
Shenghua & 194814 & 0.47719 & 47517.91 \\
\hline
\end{tabular}

Table 2. Market value of asset and asset volatility.

\begin{tabular}{ccccc}
\hline ST Company & $V_{A}$ (Ten thousand) & $\sigma_{A}$ & Non-ST Company & $V_{A}$ (Ten thousand) \\
\hline${ }^{*}$ ST Yuancheng & 148305.98 & 0.274131 & Jinma & 304425.81 \\
${ }^{*}$ ST Zhongda & 549348.01 & 0.245261 & Lianchuang & 536586.69 \\
${ }^{*}$ ST Tianrun & 151854.66 & 0.427553 & Kaile & 547666.55 \\
ST Guofa & 226851.81 & 0.350167 & Batian & 206727.72 \\
ST Luodun & 287007.76 & 0.467563 & Jiahua & 0.5058 \\
ST Tianyi & 144982.83 & 0.294551 & Shenghua & 0.339649 \\
\hline
\end{tabular}


Table 3. Default to distance.

\begin{tabular}{cccc}
\hline ST Company & $\mathrm{DD}_{1}$ & Non-ST Company & $\mathrm{DD}_{1}$ \\
\hline${ }^{*}$ ST Yuancheng & 2.476168 & Jinma & 2.374801 \\
${ }^{*}$ ST Zhongda & 2.265421 & Lianchuang & 1.947293 \\
"ST Tianrun & 1.90848 & Kaile & 2.643471 \\
ST Guofa & 2.249896 & Batian & 1.856881 \\
ST Luodun & 1.716353 & Jiahua & 2.818858 \\
ST Tianyi & 2.426656 & Shenghua & 2.111437 \\
\hline
\end{tabular}

Table 4. Default to distance of uncertainty KMV.

\begin{tabular}{cccc}
\hline ST Company & $\mathrm{DD}_{2}$ & Non-ST Company & $\mathrm{DD}_{2}$ \\
\hline "ST Yuancheng & 2.196838 & Jinma & 2.840759 \\
" ST Zhongda & 1.935192 & Lianchuang & 2.430826 \\
"ST Tianrun & 1.678772 & Kaile & 3.087181 \\
ST Guofa & 2.010186 & Batian & 2.352168 \\
ST Luodun & 1.48924 & Jiahua & 3.314398 \\
ST Tianyi & 2.163204 & Shenghua & 2.588359 \\
\hline
\end{tabular}

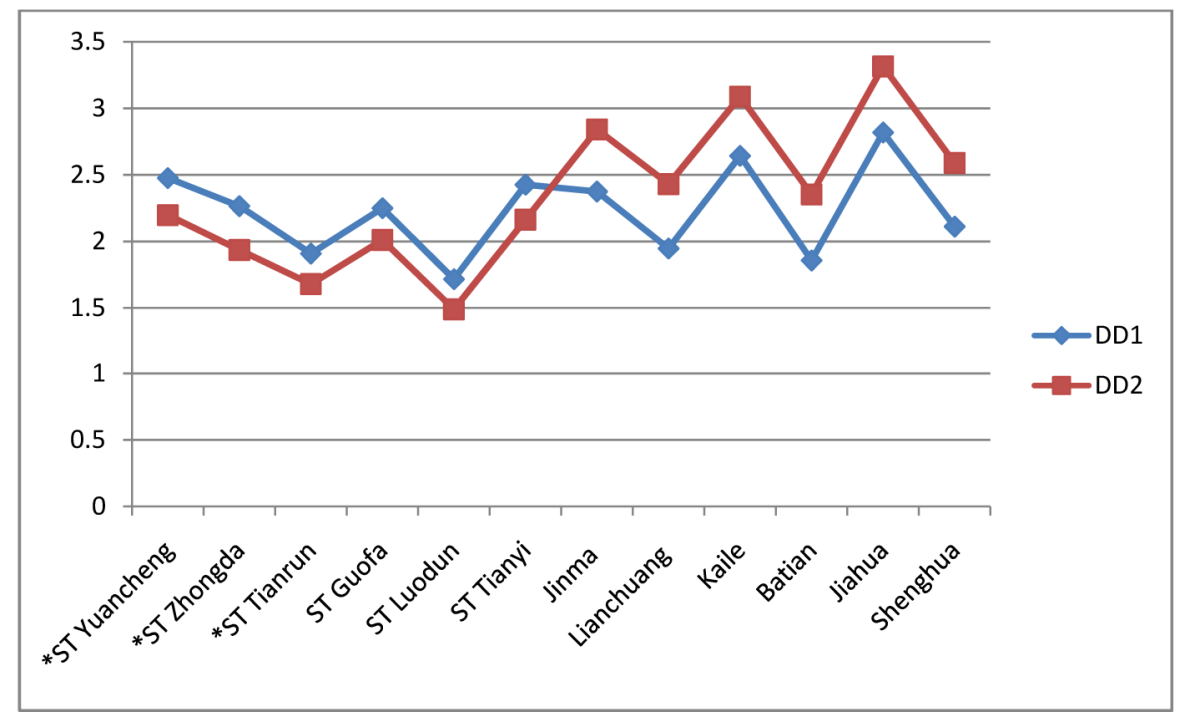

Figure 4. Comparation of $\mathrm{DD}_{1}$ and $\mathrm{DD}_{2}$.

the model is flexible and consistent with the actual situation.

\section{Conclusions}

By the comparison of the two models above, we found that the uncertain KMV model can distinguish ST and non-ST-companies better than traditional KMV model. Interest rate changes such as the uncertainty factor in the economic system, belong to the systemic risk which cannot be dispersed.

In this paper, an uncertain variable method was proposed to generate interest rate and the uncertainty distri- butions were obtained by Delphi method or assumed to be lognormal distribution. However, we will still continue to research on uncertainty interest rate.

\section{REFERENCES}

[1] W. Zhang, “A Study on Listed Company’s Credit Risk Measurement Based on KMV Model,” 2008.

[2] Z. J. Zhang, X. H. Chen and F. Q. Wang, "Research on Credit Risk of Small and Medium-Sized Listed Companies in China Based on KMV Model," Journal of Finance and Economics, Vol. 33 No. 1, 2007.

[3] X. Y. Liang, “A Study on Medium-Sized Listed Compa- 
nies' Credit Risk Measurement Based on KMV Model,” 2012.

[4] S. C. Yang, "Empirical Study on Credit Risk Measurement Methods and KMV Model," 2010.

[5] B. Liu, "Uncertainty Theory," 2nd Edition, Springer-Verlag, Berlin, 2007.

[6] B. Liu, "Uncertainty Theory: A Branch of Mathematics for Modeling Human Uncertainty,” Springer-Verlag, Berlin, 2010.

[7] S. Zeng and J. Zhou, "Structure of Default Risk, Interest Rate Fluctuations and Debt maturity-Interest Rate's Feedback Effects of Corporate Debt Financing,” Journal of Jiangxi University of Finance and Ecomomics, No. 3, 2008. 